Pacific Journal of Mathematics

QUASIMEASURES AND OPERATORS COMMUTING WITH 


\section{QUASIMEASURES AND OPERATORS COMMUTING WITH CONVOLUTION}

\section{G. I. GAUDRY}

Let $G$ be a Hausdorff locally compact abelian group. In this paper we characterise completely those continuous linear operators $T$ from $C_{c}(G)$ (the space of continuous functions with compact supports endowed with the inductive limit topology) into $M(G)$ (the space of measures with the vague topology of measures) which commute with convolution: $T(f * g)=(T f) * g$. They are represented by convolution with a "quasimeasure". As a corollary of this theorem, we have the result that the space of multipliers from $L^{p}(G)(p \neq \infty)$ to $L^{q}(G)$ is isomorphic to a subspace of the space of quasimeasures.

The quasimeasures are defined as the elements of the dual of a certain inductive limit of Banach spaces. We develop some of the theory of pseudomeasures and of quasimeasures and establish the structural relationship of quasimeasures to pseudomeasures.

Throughout, $G$ will denote a Hausdorff locally compact abelian group, $X$ its character group. $M, M_{b d}, M_{c}$ will denote the spaces of measures, bounded measures and measures with compact supports respectively. Where necessary, we shall write $M(G), M(X)$ etc. to distinguish the spaces of measures etc. over $G$ and $X$. We shall write $\varepsilon_{a}$ for the Dirac measure at the point $a$.

Several function spaces will be of importance:

$C$ will be the space of continuous complex-valued functions. $C_{c}$ with denote the space of continuous functions with compact supports, regarded topologically as the internal inductive limit of the spaces $C_{c, K}$ (the space of continuous functions with support in $K, K$ compact, and the usual sup norm topology). The support of a function $f \in C$ will be denoted $[f]$.

$L^{p}(1 \leqq p \leqq \infty)$ will be the Lebesgue spaces determined by Haar measure, the elements being equivalence classes as usual. The Haar measures $d x, d \chi$ on $G, X$ respectively will be assumed normalised so that Plancherel's Theorem holds.

$A(G)$ will denote the space of Fourier transforms of functions integrable over $X$. By virtue of the semi-simplicity of $L^{1}(X), A(G)$ is isomorphic to $L^{1}(X)$. We define the topology of $A(G)$ so as to make it a Banach algebra under pointwise multiplication as follows:

$$
\|\hat{f}\|_{A^{(}(G)}=\|f\|_{L^{1}(X)} \quad(\hat{f} \in A(G))
$$

$A_{c}(G)$ is the subspace of $A(G)$ formed of functions whose supports are 
compact. Note that $A_{c}(G)$ is dense in $A(G)$.

$f_{\vee}$ will denote the reflection of a function $f$.

In this paper, we characterise completely those linear mappings $T: C_{c} \rightarrow M$, continuous for the inductive limit topology on $C_{c}$ and the vague $\left(\sigma\left(M, C_{c}\right)\right)$ topology of measures on $M$, which commute with convolution:

$$
T(f * g)=(T f) * g \quad\left(f, g \in C_{c}\right) .
$$

In order to do this, we introduce the concept of quasimeasure. In Section 2, some of the basic properties of quasimeasures are established.

1. Pseudomeasures and their properties. The concept of pseudomeasure is already well-known (Kahane [9]). For completeness, we set down the definition and derive several important properties of pseudomeasures.

Definition 1.1. We denote by $P(G)$ the dual of $A(G)$ (with its topology as defined above). The elements of $P(G)$ are called pseudomeasures.

The Fourier transform of $\sigma \in P(G)$ is defined as follows: $\hat{\sigma}$ is the continuous linear form on $L^{1}(X)$ given by

$$
\hat{\sigma}(f)=\sigma\left(\hat{f}_{\vee}\right) \quad\left(f \in L^{1}(X)\right) .
$$

Note that $\hat{\sigma}$ can be identified with an element of $L^{\infty}(X)$. This will often be done. We define the convolution of two pseudomeasures via the Fourier transform:

$$
\left(\sigma_{1} * \sigma_{2}\right)^{\wedge}=\hat{\sigma}_{1} \cdot \hat{\sigma}_{2} .
$$

With this definition of multiplication and the topology as the strong dual of $A(G), P(G)$ is, under the Fourier transform, isometrically isomorphic to $L^{\infty}(X)$.

Note further that $M_{b d}(G) \subset P(G)$ and that we can easily define multiplication of a pseudomeasure by a Fourier-Stieltjes transform of a bounded measure:

$$
(\widehat{\mu} \sigma)(f)=\sigma(\hat{\mu} f) \quad\left(\sigma \in P(G), \mu \in M_{b d}(X), f \in A(G)\right) .
$$

It is necessary to be able to define the support of a pseudomeasure and in order to make this definition, we establish a lemma on partitions of unity for $A(G)$.

Lemma 1.2. Suppose $\left\{\Omega_{i}\right\}_{i \in I}$ is a cover of $G$ by open sets. Then 
there exists a locally finite family $\left\{f_{j}\right\}_{j \in J}$ of functions in $A_{c}(G)$ such that to each $j \in J$, there exists at least one $i \in I$ with $\left[f_{j}\right] \subset \Omega_{i}$, and such that $0 \leqq f_{j}(x) \leqq 1, \sum_{j \in J} f_{j}(x)=1$ everywhere in $G$. (If the original cover is a locally finite cover by open relatively compact sets, $J$ may be chosen the same as $I$ and the $f_{i}$ with $\left[f_{i}\right] \subset \Omega_{i}$ etc.)

Proof. $G$ is locally compact, so there exists a cover $\left\{\Omega_{i^{\prime}}^{I}\right\}_{i^{\prime} \in I^{\prime}}$ of $G$ by open relatively compact sets such that for each $i^{\prime} \in I^{\prime}$, there is at least one $i \in I$ with $\Omega_{\imath^{\prime}}^{I} \subset \Omega_{i}$. Again, every locally compact $T_{0}$ group is paracompact (Hewitt and Ross [7], Th. 8.13). Thus, we can find a locally finite cover $\left\{\Omega_{j}^{I I}\right\}_{j \in J}$ of $G$ by open sets such that, for each $j \in J$, there is at least one $i^{\prime} \in I^{\prime}$ with $\Omega_{j}^{I I} \subset \Omega_{i^{\prime}}^{I}$. Then the sets $\Omega_{j}^{I I}$ are relatively compact, and $\left\{\Omega_{j}^{I I}\right\}_{j \in J}$ is a locally finite cover of $G$ by open relatively compact sets. Now choose two further open covers of $G$, say $\left\{\Omega_{j}^{I I I}\right\}_{j \in J}$, $\left\{\Omega_{j}^{r V}\right\}_{j} \in J$ such that

$$
\Omega_{j}^{I V} \subset \overline{\Omega_{j}^{I V}} \subset \Omega_{j}^{I I I} \subset \overline{\Omega_{j}^{I I I}} \subset \Omega_{j}^{I I} \quad(j \in J) .
$$

This choice is certainly possible (Bourbaki [1], Section 4, Th. 3).

For each $j \in J$, choose $\varphi_{j} \in A^{*}(G)$ with $\varphi_{j}=1$ on $\overline{\Omega_{j}^{I V}}, \varphi_{j}=0$ outside $\Omega_{J}^{I I I}$ (Rudin [10], Th. 2.6.2). Then the family $\left\{\varphi_{j}\right\}_{j \in J}$ is locally finite. Write $f_{j}=\varphi_{j} / \Sigma \varphi_{j}\left(\Sigma \varphi_{j}>0\right.$ since $\left\{\Omega_{j}^{I V}\right\}_{j \in J}$ covers $\left.G\right)$. In order to show $f_{j} \in A_{c}(G)$ it suffices to show $1 / \Sigma \varphi_{j}$ is, on $\overline{\Omega_{j}^{I I}}$, the restriction of an element of $A(G) . \quad \overline{\Omega_{j}^{I I}}$ is compact, and $\left\{\varphi_{j}\right\}_{j \in J}$ is locally finite; so there are only a finite number, say $\varphi_{j_{1}}, \cdots, \varphi_{j_{n}}$ of members of $\left\{\varphi_{j}\right\}_{j \in J}$ which are not identically zero on $\overline{\Omega_{j}^{I I I}}$. Further, $\sum_{\nu=1}^{n} \varphi_{j_{\nu}}>0$ on $\overline{\Omega_{j}^{I I}}$, and $\Sigma \varphi_{j_{\nu}}=\varphi \in A(G)$. Thus it suffices to show that, it $K$ is a compact subset of $G$, if $\varphi \in A(G)$ and if $\varphi>0$ on $K$, then on $K, 1 / \varphi=\psi$ for some $\psi \in A(G)$.

Consider then the quotient Banach algebra $B=L^{1}(X) / I_{0}$, where $I_{0}$ is the closed ideal of functions in $L^{1}(X)$ whose transforms vanish on $K . \quad B$ is isomorphic to the algebra of restrictions to $K$ of functions in $A(G)$. Its maximal ideal space is $K$. But $\varphi>0$ on $K$, so $\varphi \mid K$ is invertible, whence the desired result.

DeFinition 1.3. We say a pseudomeasure $\sigma$ is zero on an open set $\Omega \subset G$ if $\sigma(f)=0$ for all $f \in A(G)$ with $[f] \subset \Omega$. Using 1.2 and the above definition, we define the support of a pseudomeasure.

Definition 1.4. The support of a pseudomeasure $\sigma \in P(G)$, denoted $[\sigma]$ is defined as the complement of the largest open set on which $\sigma$ vanishes.

Note that the support as defined in 1.4 coincides with the support of a continuous function as usually defined, so there is no confusion 
in the notation $[\sigma]$.

It is of interest to be able to characterise the pseudomeasures with point support, and we have the expected result:

THEOREM 1.5. If $\sigma$ is a pseudomeasure with point support $\{a\}$, then $\sigma=\lambda \varepsilon_{a}$ for some scalar $\lambda$.

Proof. Since $\sigma$ is a pseudomeasure on $G$, we have

$$
|\sigma(\hat{f})| \leqq M\|f\|_{1} \quad\left(f \in L^{1}(X)\right) .
$$

Suppose $[\sigma]=\{0\}$. Now if $\hat{f}(0)=0, \varepsilon>0$, there exists $k \in L^{1}$ with $[\hat{k}]$ in an arbitrarily small neighbourhood of $0, \hat{k}=1$ on some neighbourhood of $0,\|k\|_{1}<2$ and $\|f * k\|_{1}<\varepsilon / M$ (Rudin [10], Th. 2.6.3). Then $\sigma(\hat{f} \hat{k})=\sigma(\hat{f})$ since $[\sigma]=\{0\}$, and

$$
|\sigma(\hat{f} \hat{k})| \leqq M\|f * k\|_{1}<\varepsilon .
$$

Hence $\sigma(\hat{f})=0$.

Consider the continuous linear form on $A(G)$ defined by $\varepsilon_{0}: \varepsilon_{0}(\hat{f})=$ $\hat{f}(0)$. Write $N=\{\hat{f} \in A(G): \hat{f}(0)=0\}$, the null space of $\varepsilon_{0}$. By our above argument, $\sigma(N)=0$. Hence (Bourbaki [2], Section 4, Th. 1) $\sigma=\lambda \varepsilon_{0}$ for some scalar $\lambda$.

\section{Quasimeasures and their properties.}

Definition 2.1. Suppose $K$ a compact subset of $G$. We define $D_{K}(G)$ as the following vector space of continuous functions with its associated topology.

$$
\begin{aligned}
D_{K}(G)=\left\{u \in C_{c}(G): u=\right. & \sum_{1}^{\infty} f_{i} * g_{i}, f_{i}, g_{i} \in C_{c, K}(G) \text { and } \\
& \left.\sum_{1}^{\infty}\left\|f_{i}\right\|_{\infty}\left\|g_{i}\right\|_{\infty}<\infty\right\}
\end{aligned}
$$

$D_{K}(G)$ is normed as follows:

$$
\begin{aligned}
\|u\|_{D_{K}}=\inf \left\{\sum\left\|f_{i}\right\|_{\infty}\left\|g_{i}\right\|_{\infty}: u=\sum f_{i} * g_{i},\right. \\
\left.\qquad f_{i}, g_{i} \in C_{c, K}(G) \text { and } \sum\left\|f_{i}\right\|_{\infty}\left\|g_{i}\right\|_{\infty}<\infty\right\} .
\end{aligned}
$$

Evidently, $D_{K}(G) \subset C_{c, K+K}(G)$ and $\|u\|_{\infty} \leqq \rho_{K}\|u\|_{D_{K}}$ where $\rho_{K}$ is the measure of $K$.

DeFinition 2.2. We define $D(G)$ as the internal inductive limit of the spaces $D_{K}(G)$.

Definition 2.3. The elements of $D^{\prime}(G)$, the dual of $D(G)$, are called quasimeasures. (Thus, $s$ is a quasimeasure on $G$ if and only if $s$ is a linear form on $D(G)$ and $s \mid D_{K}(G)$ is continuous for the topology 
of $D_{K}(G)$, as defined in 2.1, for each compact subset $K$ of $G$.)

THEOREM 2.4. $D_{K}(G)$ is complete.

Proof. Suppose that $\left(u_{n}\right)_{1}^{\infty}$ is a Cauchy sequence in $D_{K}(G)$. It will be sufficient to show that a subsequence of $\left(u_{n}\right)$ converges to an element of $D_{K}(G)$. Without loss of generality then, we may suppose that $\left\|u_{n+1}-u_{n}\right\|_{D_{K}} \leqq 1 / 2^{n} \quad(n=1,2, \cdots)$. Write $\left\|u_{1}\right\|_{D_{K}}=C$. We may also write the following expansions:

$$
\begin{gathered}
u_{1}=\sum_{k=1}^{\infty} f_{1 k} * g_{1 k} \\
u_{n+1}-u_{n}=\sum_{k=1}^{\infty} f_{n+1, k} * g_{n+1, k} \quad(n=1,2, \cdots)
\end{gathered}
$$

with

$$
\sum_{k=1}^{\infty}\left\|f_{1 k}\right\|_{\infty}\left\|g_{1 k}\right\|_{\infty}<C+1
$$

and

$$
\sum_{k=1}^{\infty}\left\|f_{n+1, k}\right\|_{\infty}\left\|g_{n+1, k}\right\|_{\infty}<\frac{1}{2^{n-1}} \quad(n=1,2, \cdots) .
$$

Define

$$
u=f_{11} * g_{11}+f_{12} * g_{12}+f_{21} * g_{21}+f_{31} * g_{31}+\cdots .
$$

Clearly, $u \in D_{K}(G)$ since

$$
\left\|f_{11}\right\|_{\infty}\left\|g_{11}\right\|_{\infty}+\left\|f_{12}\right\|_{\infty}\left\|g_{12}\right\|_{\infty}+\cdots<C+3 \text {. }
$$

We now show that $u_{n} \rightarrow u$ in $D_{K}$. Given $\varepsilon>0$, choose a natural number $n_{0}$ such that $\sum_{r=n}^{\infty} 1 / 2^{r-1}<\varepsilon\left(n>n_{0}\right)$; if $n>n_{0}$, then

$$
\begin{aligned}
u-u_{n+1} & =u-\left[\left(u_{n+1}-u_{n}\right)+\cdots+\left(u_{2}-u_{1}\right)+u_{1}\right] \\
& =f_{n+2,1} * g_{n+2,1}+f_{n+2,2} * g_{n+2,2}+f_{n+3,1} * g_{n+3,1}+\cdots
\end{aligned}
$$

and

$$
\left\|u-u_{n+1}\right\|_{D_{K}}<\sum_{r=n+1}^{\infty} \frac{1}{2^{r-1}}<\varepsilon .
$$

So $u_{n} \rightarrow u$ in $D_{K}(G)$.

We may now prove the following theorem.

TheOREM 2.5. $D(G) \subset A_{c}(G), D(G)$ is dense in $A_{c}(G)$, hence is dense in $A(G)$, and the topology of $D(G)$ is stronger than that induced by $A(G)$. 
Proof. Suppose that $u \in D_{K}(G), u=\sum f_{i} * g_{i}, f_{i}, g_{i} \in C_{c, \boldsymbol{K}}$ and $\sum\left\|f_{i}\right\|_{\infty}\left\|g_{i}\right\|_{\infty}<\infty$. Write $s_{n}=\sum_{1}^{n} f_{i} * g_{i}$, so that $s_{n} \in D_{K}(G)$, and $s_{n} \rightarrow u$ in $D_{K}(G)$. Also $s_{n} \rightarrow u$ uniformly.

It follows from Theorem 1.6.3 of Rudin [10] that $s_{n} \in A(G)$. Now

$$
\begin{aligned}
\left\|s_{m}-s_{n}\right\|_{A^{(G)}} & =\left\|\sum_{n+1}^{m} \hat{f}_{i} \hat{g}_{i}\right\|_{L^{1}(X)} \leqq \sum_{n+1}^{m}\left\|\hat{f}_{i} \hat{g}_{i}\right\|_{L^{1}(X)} \\
& \leqq \sum_{n+1}^{m}\left\|\hat{f}_{i}\right\|_{2}\left\|\hat{g}_{i}\right\|_{2} \quad \text { (by Hölder's inequality) } \\
& =\sum_{n+1}^{m}\left\|f_{i}\right\|_{2}\left\|g_{i}\right\|_{2} \quad \text { (Plancherel's Theorem) } \\
& \leqq \lambda_{K} \sum_{n+1}^{m}\left\|f_{i}\right\|_{\infty}\left\|g_{i}\right\|_{\infty}
\end{aligned}
$$

where $\lambda_{K}$ is a constant. So $\left\|s_{m}-s_{n}\right\|_{A^{(\theta)}} \leqq \lambda_{K} \sum_{n+1}^{m}\left\|f_{i}\right\|_{\infty}\left\|g_{i}\right\|_{\infty}$ and $\left(s_{n}\right)_{1}^{\infty}$ is a Cauchy sequence in $A(G)$. Hence $s_{n} \rightarrow v$ say in $A(G)$ since $A(G)$ is complete; so $s_{n} \rightarrow v$ uniformly. Hence $u=v$. Since the elements of $D(G)$ have compact supports, we have shown that $D(G) \subset A_{c}(G)$. Further, $\quad\|u\|_{A^{(}(G)}=\lim \left\|s_{n}\right\|_{A^{\prime}(G)} \leqq \lambda_{K} \sum_{1}^{\infty}\left\|f_{i}\right\|_{\infty}\left\|g_{i}\right\|_{\infty}$, and we have then that $\|u\|_{A^{(\theta)}} \leqq \lambda_{K}\|u\|_{D_{K^{(}(\theta)}}$ : this implies that the topology of $D(G)$ is stronger than that induced by $A(G)$.

To prove that $D(G)$ is dense in $A_{c}(G)$, suppose that $f \in A_{c}(G)$ with $[f]=K$, a compact set. Write $\left(\varphi_{\beta}\right)$ for an approximate identity in $L^{1}(G)$ with $\left\|\varphi_{\beta}\right\|_{1}=1, \varphi_{\beta} \in C_{c, K_{0}}$ for all $\beta$ and consider $f-\varphi_{\beta} * f$. Note that $\varphi_{\beta} * f \in D(G)$ since $\varphi_{\beta}, f \in C_{c}(G)$. Then since $\hat{f} \in L^{1}(X)$, $\left\|\hat{\varphi}_{\beta}\right\|_{\infty} \leqq 1$, and $\hat{\varphi}_{\beta} \rightarrow 1$ uniformly on compact sets we have that $\left\|f-\varphi_{\beta} * f\right\|_{A(\theta)}=\left\|\hat{f}-\hat{\varphi}_{\beta} \hat{f}\right\|_{L^{1}(X)}$ tends to zero. This completes the proof of the theorem.

CoRollary 2.6. $D(G)$ is a dense vector subspace of $C_{c}(G)$ and if $f \in C_{c, \boldsymbol{K}}$, then $f=\lim u_{\beta}$ where $u_{\beta} \in D_{K_{0}}$ for some fixed $K_{0}$.

Corollary 2.7. $M(G) \subset D^{\prime}(G)$ and $P(G) \subset D^{\prime}(G)$.

We now prove a theorem which gives a characterisation of those quasimeasures which are measures.

THEOREM 2.8. A quasimeasure $s$ is a measure if and only if for every compact subset $K$ of $G, s \mid D_{K}(G)$ is continuous for the supnorm topology on $D_{K}(G)$.

Proof. The necessity is obvious. The sufficiency may be proved as follows: if $s \mid D_{K}(G)$ is continuous for the sup-norm topology, then $s \mid D_{K}(G)$ has a unique continuous extension to the closure $\overline{D_{K}(G)}$ of 
$D_{K}(G)$ in $C_{c, K+K}(G)$. Now $\bigcup_{K} \overline{D_{K}(G)}=C_{c}(G)$ by Corollary 2.6. We have only to show that if $\varphi \in C_{c}(G)$ and if $\varphi=\lim u_{n}$ with $u_{n} \in D_{K_{1}}(G)$, and $\varphi=\lim v_{n}$ with $v_{n} \in D_{K_{2}}(G)$, then $\lim s\left(u_{n}\right)=\lim s\left(v_{n}\right)$. Without loss of generality, suppose that $0 \in K_{i n} K_{2}$. Then $u_{n} \in D_{K_{1}+K_{2}}(G)$ and $v_{n} \in D_{K_{1}+K_{2}}(G)$ for all $n$. It follows immediately that $s\left(u_{n}-v_{n}\right) \rightarrow 0$ since $u_{n}-v_{n} \rightarrow 0$ uniformly.

The definition of the convolution of a quasimeasure and a function in $C_{c}(G)$ proceeds in the natural way via the tensor product. In order to show this again gives a quasimeasure, we prove:

Lemma 2.9. For a given $f \in C_{c}(G)$, the mapping $T: g \rightarrow f_{\vee} * g$ is continuous from $D(G)$ into $D(G)$.

Proof. Since $g \in D(G), g \in C_{c}(G)$ and $f_{\vee} * g \in D(G) . \quad T$ thus maps into $D(G)$.

To see that $T$ is continuous, observe that if we restrict $T$ to a given $D_{K}(G)$, the restriction is certainly continuous. For suppose that $g_{i} \rightarrow g$ in $D_{K}(G)$; then $g_{i} \rightarrow g$ uniformly over $K+K$, and $f_{\vee} * g_{i} \rightarrow f_{\vee} * g$ in $D(G)$.

The proof is now complete.

Note next that $f_{\vee} * g(\xi)=\int f(\eta) g(\xi+\eta) d \eta$ for $f, g \in C_{c}(G)$; so it is natural to define $s * f \in D^{\prime}(G)$ as follows

$$
s * f(g)=s\left(f_{\vee} * g\right) \quad(g \in D(G)) .
$$

We now study the continuity of the map $f \rightarrow s * f$. The result we obtain will be improved in Section 3 .

Lemma 2.10. The mapping $f \rightarrow s * f$ from $C_{c}(G)$ into $D^{\prime}(G)$ is continuous for the inductive limit topology on $C_{c}(G)$ and the weak $\sigma\left(D^{\prime}, D\right)$ topology on $D^{\prime}(G)$.

Proof. Suppose $g \in D(G),\left(f_{i}\right)$ a net converging to zero in $C_{c, K}$ ( $K$ a fixed compact subset of $G$ ). Write $K^{\prime}=(-K) \cup\{0\}, K_{0}=$ $K^{\prime}+([g] \cup\{0\})$. Then $f_{i \vee} * g \in D_{K_{0}}$ and $f_{i \vee} * g \rightarrow 0$ in $D_{K_{0}}$. So $s * f_{i}(g) \rightarrow 0$. Thus the restriction of the map $f \rightarrow s * f$ to each $C_{c, K}$ is continuous, and it follows that the mapping $f \rightarrow s * f$ is continuous from $C_{c}(G)$ into $D^{\prime}(G)$.

As for measures and pseudomeasures, we need the concept of the support of a quasimeasure. For this we need: 
Lemma 2.11. If $\mu \in M_{b d}(X)$, and $u=\sum f_{i} * g_{i}$ is an element of $D_{K}(G)$, then

$$
H=\int_{X} \chi^{-1} u d \mu(\chi)=\sum \int_{X}\left(\chi^{-1} f_{i}\right) *\left(\chi^{-1} g_{i}\right) d \mu(\chi)
$$

is an element of $D_{K}(G)$, and for all $x \in G$,

$$
H(x)=\sum \hat{\mu}(x) \cdot f_{i} * g_{i}(x)=\hat{\mu}(x) u(x) .
$$

(Note that the integrals appearing here are vector-valued integrals.)

Proof. It is clear that $\chi^{-1} u=\sum\left(\chi^{-1} f_{i}\right) *\left(\chi^{-1} g_{i}\right)$ is an element of $D_{K}(G)$. Consider then the mapping $\chi \rightarrow \chi^{-1} u$ from $X$ into $D_{K}(G)$. This mapping is certainly uniformly bounded; we show that it is, in addition, continuous. If $\chi, \chi_{0} \in X$, then

$$
\left(\chi^{-1} f_{i}\right) *\left(\chi^{-1} g_{i}\right)-\left(\chi_{0}^{-1} f_{i}\right) *\left(\chi_{0}^{-1} g_{i}\right)
$$

is an element of $D_{K}(G)$, and

$$
\begin{aligned}
& \left\|\left(\chi^{-1} f_{i}\right) *\left(\chi^{-1} g_{i}\right)-\left(\chi_{0}^{-1} f_{i}\right) *\left(\chi_{0}^{-1} g_{i}\right)\right\|_{D_{K}} \\
& \leqq\left\|\left(\chi^{-1}-\chi_{0}^{-1}\right) f_{i}\right\|_{\infty}\left\|g_{i}\right\|_{\infty}+\left\|f_{i}\right\|_{\infty}\left\|\left(\chi^{-1}-\chi_{0}^{-1}\right) g_{i}\right\|_{\infty} .
\end{aligned}
$$

It follows that

$$
\left\|\chi^{-1} u-\chi_{0}^{-1} u\right\|_{D_{K}} \leqq \sum\left[\left\|\left(\chi-\chi_{0}\right) f_{i}\right\|_{\infty}\left\|g_{i}\right\|_{\infty}+\left\|f_{i}\right\|_{\infty}\left\|\left(\chi-\chi_{0}\right) g_{i}\right\|_{\infty}\right] .
$$

But $\chi \rightarrow \chi_{0}$ in $X$ if and only if $\chi \rightarrow \chi_{0}$ uniformly on compact subsets of $G$; and $f_{i}, g_{i} \in C_{c, K}(G)$; so if $\chi \rightarrow \chi_{0}, \chi^{-1} u \rightarrow \chi_{0}^{-1} u$ in $D_{K}(G)$, and we have established the continuity of the map $\chi \rightarrow \chi^{-1} u$ from $X$ into $D_{K}(G)$.

The measure $\mu$ is bounded and $D_{K}(G)$ is complete. Hence $\int_{x} \chi^{-1} u d \mu(\chi)$ is an element of $D_{K}(G)$ (Edwards [4], (8.14.14)). The series for $\chi^{-1} u$ is uniformly convergent in $D_{K}(G)$ and its partial sums are uniformly bounded. The second assertion of (2.11.1) follows.

(2.11.2) follows since the evaluation functionals are continuous on $D_{K}(G)$ (see Edwards [4], 8.14.1). This completes the proof of the theorem.

DeFinition 2.12. We say a quasimeasure $s$ vanishes on an open subset $\Omega$ of $G$ if $s(f)=0$ for all $f \in D(G)$ with support in $\Omega$. We say two quasimeasures are equal on $\Omega$ if their difference vanishes on $\Omega$.

LEMMA 2.13. If $\left\{\Omega_{i}\right\}$ is a family of open subsets of $G$ and if $s=0$ on $\Omega_{i}$ for all $i$, then $s=0$ on $\Omega=\bigcup_{i} \Omega_{i}$. 
Proof. Suppose $f \in D(G)$ with $[f] \subset \Omega$. Since $[f]=K$ is compact, there exists a finite number of members of $\left\{\Omega_{i}\right\}$, say $\Omega_{i_{1}}, \cdots, \Omega_{i_{n}}$ which form a cover of $K$. Then, by Lemma 1.2, there exist $f_{1}, \cdots, f_{n} \in A(G)$ with $\left[f_{j}\right] \subset \Omega_{i_{j}}, f_{j} \geqq 0,(j=1, \cdots, n)$ and $\sum_{1}^{n} f_{j}=1$ on $K$.

Since $f_{j}$ has the form $\int_{X} \chi^{-1} d \mu(\chi)$ for some $\mu \in M_{b d}(X)$ and $f \in D_{K_{0}}(G)$ for some $K_{0}$, Lemma 2.11 implies that $f_{j} f \in D_{K_{0}}(G) \subset D(G)$ and $\left[f_{j} f\right] \subset \Omega_{i_{j}}$. Hence $s\left(f_{j} f\right)=0$ since $s=0$ on $\Omega_{i_{j}}$. But $f=\sum_{1}^{n}\left(f_{j} f\right)$ so $s(f)=0$ and we have, since $f$ was arbitrary, $s=0$ on $\Omega$.

Now we are in a position to make our definition.

DeFinition 2.14. The support of a quasimeasure is the complement relative to $G$ of the largest open set on which it vanishes.

REMARK. It is easy to see that for pseudomeasures, this definition of support coincides with that given in 1.4. Similarly for measures, the usual definition and the new definition of support coincide. We shall write $[s]$ for the support of a quasimeasure $s$.

Later we shall need to be able to write down a quasimeasure which has compact support, and which is equal to a given quasimeasure on a given open relatively compact set. To be able to do this, we define what is meant by $\hat{\mu} s$ for $\mu \in M_{b d}(X), s \in D^{\prime}(G)$.

LemMa 2.15. If $\mu \in M_{b d}(X)$, the mapping $u \rightarrow \hat{\mu} u$ is continuous from $D_{K}(G)$ into $D(G)$.

Proof. By Lemma 2.11, the mapping is certainly into $D(G)$. If $u \in D_{K}(G)$ and $\chi \in X$, then $\left\|\chi^{-1} u\right\|_{D_{K}} \leqq\|u\|_{D_{K}}$. So,

$$
\begin{aligned}
\|\hat{\mu} u\|_{D_{K}} & =\left\|\int\left(\chi^{-1} u\right) d \mu(\chi)\right\|_{D_{K}} \\
& \leqq \int\left\|\chi^{-1} u\right\|_{D_{K}} d|\mu|(\chi) \\
& \leqq\|u\|_{D_{K}}\|\mu\| .
\end{aligned}
$$

Continuity follows immediately.

We can now make our definition.

Definition 2.16. If $\mu \in M_{b d}(X), s \in D^{\prime}(G)$, we define $\hat{\mu} s \in D^{\prime}(G)$ by

$$
\widehat{\mu} s(u)=s(\hat{\mu} u) \quad(u \in D(G)) .
$$

Some of the most important properties of quasimeasures, in particular their structural relationship with pseudomeasures, are 
deduced in Section 4 as corollaries of theorems proved in Section 3 and other known results.

3. Operators commuting with convolution. The problem of characterising those continuous linear maps from one space to another which commute with translations or convolution is well-known. (See for example Edwards [3], [5], Hörmander [8].) We give a complete characterisation in terms of convolution with quasimeasures, of those linear operators $T: C_{c} \rightarrow M$ which are continuous for the natural (inductive limit) topology on $C_{c}$ and the vague topology of measures on $M$, and which commute with convolution. For brevity, we shall call such operators $T$ multipliers from $C_{c}$ into $M$. We first prove a useful lemma.

LEMma 3.1. Suppose that $T$ is a multiplier from $C_{c}$ into $M$. Then there exists a net $\left(T_{\beta}\right)$ of multipliers from $C_{c}$ into $M$ with the following properties:

(i) Each $T_{\beta}$ is defined by a measure $\mu_{\beta}$ :

$$
T_{\beta} f=\mu_{\beta} * f \quad\left(f \in C_{c}\right) .
$$

(ii) If $K$ is compact subset of $G$, there exists a constant $C_{K}>0$ independent of $\beta$ such that

$$
\left|\left(T_{\beta} f\right) * g(0)\right| \leqq c_{K}\|f\|_{\infty}\|g\|_{\infty} \quad\left(f, g \in C_{c, K}\right) .
$$

(iii) If $f, g \in C_{c},\left(T_{\beta} f\right) * g(0) \rightarrow(T f) * g(0)$.

Proof. Write $\left(h_{\beta}\right)$ for an approximate identity in $L^{1}(G)$, each $h_{\beta}$ being continuous and with support in a fixed compact set $K_{0}$ and with $\left\|h_{\beta}\right\|_{1}=1$. Define $T_{\beta} f=h_{\beta} *(T f)\left(f \in C_{c}\right)$. Since $T$ commutes with convolution, and $h_{\beta} \in C_{c}$, we have $T_{\beta} f=\left(T h_{\beta}\right) * f=\mu_{\beta} * f$ say. This establishes (i).

Now each $T_{\beta}$ is linear, commutes with convolution, and maps $C_{c}$ into $M$. If $g \in C_{c}$,

$$
\left\langle T_{\beta} f, g\right\rangle=h_{\beta} * T f * g_{\vee}(0)=\left\langle T f,\left(h_{\beta} * g_{\vee}\right)_{\vee}\right\rangle
$$

and $h_{\beta} * g_{\vee} \in C_{c}$. Hence $T_{\beta}$ is continuous, and each $T_{\beta}$ is a multiplier from $C_{c}$ into $M$.

Suppose next that $K$ is a fixed compact set, and consider the mapping $f \rightarrow \int_{K} d|T f|$ from $C_{c}$ into $R$. The mapping $f \rightarrow \int_{K} d|T f|$ is a lower semi-continuous semi-norm on $C_{c}$, hence is continuous since $C_{c}$ is barrelled. So in particular, if $K$ is compact, $f \rightarrow \int_{-K} d|T f|$ is a 
continuous semi-norm on $C_{c, K}$, and there exists a constant $\lambda_{K}>0$ with $\int_{-K} d|T f| \leqq \lambda_{K}\|f\|_{\infty}\left(f \in C_{c, K}\right)$. Thus, if $f, g \in C_{c, K}$,

$$
\begin{aligned}
|(T f) * g(0)| & =\left|\int_{G} g(-y) d T f(y)\right| \\
& \leqq \int_{-K}|g(-y)| d|T f(y)| \\
& \leqq\|g\|_{\infty} \int_{-K} d|T f| \\
& \leqq \lambda_{K}\|f\|_{\infty}\|g\|_{\infty} .
\end{aligned}
$$

In order to prove (ii), we have, for $f, g \in C_{c, K}$,

$$
\begin{aligned}
\left|\left(T_{\beta} f\right) * g(0)\right| & =\left|h_{\beta} *(T f) * g(0)\right| \\
& =\left|(T f) *\left(h_{\beta} * g\right)(0)\right|
\end{aligned}
$$

and $h_{\beta} * g \in C_{c, K^{\prime}}$ say, where $K \subset K^{\prime}$ and $K^{\prime}$ is independent of $\beta$. By (3.1.2),

$$
\begin{aligned}
\left|\left(T_{\beta} f\right) * g(0)\right| & \leqq c_{K}\|f\|_{\infty}\left\|h_{\beta} * g\right\|_{\infty} \\
& \leqq c_{K}\|f\|_{\infty}\|g\|_{\infty}
\end{aligned}
$$

where $c_{K}$ is a constant independent of $\beta$. This completes the proof of (ii).

(iii) follows immediately once we note that, $g$ being in $C_{c}$, the functions $h_{\beta} * g$ are continuous and have their supports in a fixed compact set independent of $\beta$, and $h_{\beta} * g \rightarrow g$ uniformly. Then (3.1.1) gives the desired result.

We now proceed to prove our representation theorem.

Theorem 3.2. (i) Suppose that $T$ is a multiplier from $C_{c}$ into $M$. Then there exists a quasimeasure $s$ with

$$
T f=s * f \quad\left(f \in C_{c}\right)
$$

(ii) Conversely, suppose $s \in D^{\prime}$, and define the operator $T_{s}: T_{s} f=s * f\left(f \in C_{c}\right)$. Then $T_{s}$ maps $C_{c}$ into $M$ and is a multiplier from $C_{c}$ into $M$.

Proof. (i) Suppose that $T$ is a multiplier from $C_{c}$ into $M$. If $u=\sum f_{i} * g_{i}$ with $f_{i}, g_{i} \in C_{c, K}, \sum\left\|f_{i}\right\|_{\infty}\left\|g_{i}\right\|_{\infty}<\infty$ is an element of $D_{K}$, it follows immediately from (3.1.2) that $\Sigma\left(T f_{i}\right) * g_{i}(0)$ converges. We define $t(u)=\sum\left(T f_{i}\right) * g_{i}(0)$. This is a meaningful definition only if $\sum f_{i} * g_{i}=0$ and $\sum\left\|f_{i}\right\|_{\infty}\left\|g_{i}\right\|_{\infty}<\infty$ with $f_{i}, g_{i} \in C_{c, K}$ together imply that $\Sigma\left(T f_{i}\right) * g_{i}(0)=0$. Choose a net $\left(T_{\beta}\right)$ of multipliers satisfying conditions (i)-(iii) of Lemma 3.1, and consider, for each $\beta$, the series 
$s_{\beta}=\sum_{i}\left(T_{\beta} f_{i}\right) * g_{i}(0)$. We have that

$$
\begin{aligned}
\sum_{i}\left|\left(T_{\beta} f_{i}\right) * g_{i}(0)\right| & \leqq \sum_{i} c_{K}\left\|f_{i}\right\|_{\infty}\left\|g_{i}\right\|_{\infty} \\
& =c_{K} \sum_{i}\left\|f_{i}\right\|_{\infty}\left\|g_{i}\right\|_{\infty} .
\end{aligned}
$$

Thus, the series $s_{\beta}$ are convergent, uniformly with respect to $\beta$. But by property (iii) of Lemma 3.1, $T_{\beta} f_{i} * g_{i}(0) \rightarrow T f_{i} * g_{i}(0)$ for each $i$. Hence $\lim _{\beta} \sum_{i}\left(T_{\beta} f_{i}\right) * g_{i}(0)=\sum_{i}\left(T f_{i}\right) * g_{i}(0)$. Now each $T_{\beta}$ is defined by a measure $\mu_{\beta}$ and

$$
\left(T_{\beta} f_{i}\right) * g_{i}(0)=\mu_{\beta} * f_{i} * g_{i}(0)=\int_{-(K+K)} f_{i} * g_{i}(-y) d \mu_{\beta}(y) .
$$

The series $\sum f_{i} * g_{i}$ is a uniformly convergent series of continuous functions each with support contained in $K+K$. It follows that

$$
\begin{aligned}
\sum_{i} T_{\beta} f_{i} * g_{i}(0) & =\sum_{i} \int f_{i} * g_{i}(-y) d \mu_{\beta}(y) \\
& =\int\left[\sum_{i} f_{i} * g_{i}(-y)\right] d \mu_{\beta}(y) \\
& =0
\end{aligned}
$$

since $\sum f_{i} * g_{i}=0$. We have then that $\sum\left(T f_{i}\right) * g_{i}(0)=0$, and $t$ is well-defined. $t$ is clearly a linear form on $D$, and from (3.1.2),

$$
\left|\sum_{i}\left(T f_{i}\right) * g_{i}(0)\right| \leqq \lambda_{K} \sum_{i}\left\|f_{i}\right\|_{\infty}\left\|g_{i}\right\|_{\infty} \quad\left(u \in D_{K}\right) .
$$

Hence $|t(u)| \leqq \lambda_{K}\|u\|_{D_{K}}$ and $t \mid D_{K}$ is a continuous linear form on $D_{K}$ for each compact subset $K$ of $G$, i.e., $t$ is a quasimeasure. We now show that $T f=t_{\vee} * f\left(f \in C_{c}\right)$.

From (2.9.1), if $f \in C_{c}(G)$ and $g \in D(G)$,

$$
\begin{aligned}
t * f(g) & =t\left(f_{\vee} * g\right) \\
& =\left(T f_{\vee}\right) * g(0) \\
& =\int g(-y) d\left(T f_{\vee}\right) \\
& =\left(T f_{\vee}\right)\left(g_{\vee}\right) .
\end{aligned}
$$

Hence $t * f=\left(T f_{\vee}\right)_{\vee}$ where $\left(T f_{\vee}\right)_{\vee}(g)=\left(T f_{\vee}\right)\left(g_{\vee}\right)$, or equivalently, $\left(t * f_{\vee}\right)_{\vee}=T f$. But, by easy manipulations of convolutions and reflections, $\left(t * f_{\vee}\right)_{\vee}(g)=\left(t_{\vee} * f\right)(g)$, and we get finally that $T f=t_{\vee} * f\left(f \in C_{c}\right)$ by the denseness of $D$ in $C_{c}$.

(ii) The mapping $T_{s}$ clearly commutes with convolution. Firstly, we know that $s * f \in D^{\prime}$ if $f \in C_{c}$. In order to establish that $s * f \in M$, all we need do is show that $s * f \mid D_{K}$ is continuous on $D_{K}$ for the topology induced by $C_{c, K+K}$. For $g \in D, s * f(g)=s\left(f_{\vee} * g\right)$. If $g_{i} \in D_{K}$ 
and $g_{i} \rightarrow 0$ uniformly over $K+K$, then $f_{\vee} * g_{i} \rightarrow 0$ in $D_{K^{\prime}}$ say, for some compact $K^{\prime}$. Hence $s\left(f_{\vee} * g_{i}\right)=s * f\left(g_{i}\right) \rightarrow 0$ and $s * f \in M$.

Continuity of $T_{s}$. We show firstly that

$$
s * f(g)=s\left(f_{\vee} * g\right) \quad\left(f, g \in C_{c}\right) .
$$

By Corollary 2.6, we have that if $g \in C_{c}$, then $g=\lim g_{i}$ in $C_{c, K_{0}}$ for some compact $K_{0}$, where $g_{i} \in D$. But $s * f \in M$, so $s * f\left(g_{i}\right) \rightarrow s * f(g)$. Also, since $g_{i} \rightarrow g$ in $C_{c, K_{0}}, f_{\vee} * g_{i} \rightarrow f_{\vee} * g$ in $D$. Hence $s\left(f_{\vee} * g_{i}\right) \rightarrow s\left(f_{\vee} * g\right)$ and we have (3.2.2).

In order to show that the mapping $f \rightarrow s * f$ is continuous, we need only show that its restriction to each $C_{c, K}$ is continuous. By virtue of $(3.2 .2)$, this is evident.

Note. It follows from (3.2.2) and the definition of $D(G)$, that the space of multipliers from $C_{c}$ into $M$ is isomorphic to the space of quasimeasures over $G$, both being regarded as vector spaces.

4. Structural properties of quasimeasures. All the results of this section depend directly on Theorem 2 of Edwards [5]. We state this as:

THEOREM 4.1. The continuous linear operators $T$ from $C_{c}$ (inductive limit topology) into $M_{c}$ (vague topology of measures) which commute with convolution are precisely those of the form

$$
T f=s * f
$$

where $s$ is a pseudomeasure with compact support.

Note. Theorem 2 of Edwards [5] is stated in terms of operators commuting with translations. 4.1 is equivalent to the theorem in its original form since operators $T$, continuous from $C_{c}$ into $M_{c}$, commute with convolution if and only if they commute with translations.

Form Theorem 4.1 we deduce immediately:

THEOREM 4.2. Every quasimeasure with compact support is a pseudomeasure.

Proof. If $s$ is a quasimeasure with compact support, consider the mapping $T_{s}: T_{s}(f)=s * f\left(f \in C_{c}\right)$ from $C_{c}$ into $M$. In fact $T_{s}$ is linear, maps $C_{c}$ into $M_{c}$ and commutes with convolution. By Theorem 3.2, it is continuous. Again, by Theorem 4.1

$$
T_{s}(f)=t * f=s * f \quad\left(f \in C_{c}\right)
$$


where $t$ is a pseudomeasure with compact support. This implies $s=t$. For if $f, g \in C_{c}$

$$
s * f(g)=s\left(f_{\vee} * g\right)=t\left(f_{\vee} * g\right)
$$

and if $s \neq t$, we can choose $\varphi=f_{\vee} * g \in D$ with $s(\varphi) \neq t(\varphi)$. This is clearly impossible by (4.2.2) and hence $s=t$.

COROLlaRY 4.3. Every quasimeasure with point support is a scalar multiple of the Dirac measure at that point.

Proof. This follows from Theorem 1.5.

The principal structure theorem follows almost immediately from Theorem 4.2.

THEOREM 4.4. (i) Every quasimeasure is a locally finite sum of pseudomeasures (with compact supports).

(ii) Conversely, if $T$ is a locally finite sum of pseudomeasures, then $T$ is a quasimeasure.

Proof. (i) Let $\left\{\Omega_{i}\right\}_{i \in I}$ be a locally finite cover of $G$ by open relatively compact sets, and $\left\{f_{i}\right\}_{i \in I}$ a partition of unity subordinated to this cover as described in Lemma 1.2.

If $s \in D^{\prime}(G), f_{i} s \in D^{\prime}(G)$, and $\left[f_{i} s\right]$ is compact and contained in $\Omega_{i}$. Write $\sigma_{i}=f_{i} s$ where $\sigma_{i}$ is the corresponding pseudomeasure (Theorem 4.2). Then $s=\sum_{i \in I} \sigma_{i}$ and the sum is locally finite since the family $\left\{f_{i}\right\}_{i \in I}$ is.

(ii) The proof of the converse follows from the fact (established during the proof of Theorem 2.5) that if $K$ is a compact subset of $G$, then the topology of $D_{K}(G)$ is stronger than that induced by $A(G)$.

REMARK. Theorem 4.4 should be compared with Théorème XXI, Chapitre 3, of Schwartz [11].

Finally, we show that the apparently weak hypothesis that a continuous linear operator, commuting with convolution, maps $C_{c}$ into $M$ implies the much stronger result that it maps $L_{c}^{2}$ into $L_{l o c}^{2}$, where $L_{c}^{2}$ and $L_{l o c}^{2}$ are the spaces of square-integrable functions vanishing a.e. outside compact sets and locally square-integrable functions respectively.

THEOREM 4.5. Suppose $T$ is a multiplier from $C_{c}$ into $M$. Then $T$ can be extended to map $L_{c}^{2}$ into $L_{l o c}^{2}$.

Proof. Let $s$ be the quasimeasure defined by $T$ as in Theorem 
3.2. For $f \in L_{c}^{2}$ we define $T f=s * f=\sum \sigma_{i} * f$ where $s=\sum \sigma_{i}$ is an expression of $s$ as a locally finite sum of pseudomeasures. Then $T f$ is a locally finite sum of pseudomeasures, hence (Theorem 4.4) a quasimeasure. We show that $s * f \in L_{l o c}^{2}$ if $f \in L_{c}^{2}$.

Suppose $f \in L_{c}^{2}$ and that $f=0$ a.e. outside $A$, where $A$ is an open relatively compact subset of $G$. Let $B$ be any open relatively compact subset of $G$. We show that $s * f \mid B \in L^{2}(B)$. This will clearly imply that $s * f \in L_{l o c}^{2}$.

The behaviour of $s * f$ on $B$ is unaffected by the behaviour of $s$ outside $(-A)+B$. For if $s=s^{\prime}$ on $(-A)+B$, we can show that $s * f=s^{\prime} * f$ on $B$. In order to do this, suppose $g \in D(G)$ with $[g] \subset B$; then $\left[f_{v} * g\right] \subset(-A)+B$ and $s * f(g)=s\left(f_{\vee} * g\right)=s^{\prime}\left(f_{\vee} * g\right)=s^{\prime} * f(g)$. Hence $s * f=s^{\prime} * f$ on $B$.

Now choose $\varphi \in A(G)$ with $\varphi=1$ on $(-A)+B$ and $[\varphi]$ compact (Rudin [10], Th. 2.6.2). Then $\varphi s \in D^{\prime}(G)$ and $s=\varphi s$ on $(-A)+B$. Hence $s * f=(\varphi s) * f$ on $B$. But $(\varphi s) * f \in L^{2}(G)$ since $\varphi s$ is a pseudomeasure, $f \in L_{c}^{2}$, and $(\varphi s * f)^{\wedge}=(\varphi s)^{\wedge} \hat{f} \in L^{2}(X)$. Thus $s * f \mid B \in L^{2}(B)$, and the theorem is proved.

5. Multipliers of type $(p, q)$. Suppose that $p, q \in[1, \infty]$ and denote by $L_{p}^{q}$ the linear space of bounded operators from $L^{p}(G)$ into $L^{q}(G)$ which commute with translations. The elements of $L_{p}^{q}$ are called multipliers of type $(p, q)$. Hörmander [8] has shown that if $G=R^{n}$ and $\infty>p>q$, then $L_{p}^{q}=\{0\}$ while if $p \leqq q$ and $p \neq \infty, L_{p}^{q}$ is isomorphic to a certain space of tempered distributions, the operator $T$ corresponding to the tempered distribution $d$ being defined by

$$
T f=d * f \quad(f \in S)
$$

where $S$ is the space of rapidly-decreasing $C^{\infty}$ functions.

For a general LCA group $G$, Hörmander's proof goes through without change to show that $L_{p}^{q}=\{0\}$ if $\infty>p>q$ and $G$ is noncompact. We shall now show, as a corollary of Theorem 3.2, that his characterisation of $L_{p}^{q}$ for $p \leqq q$ can be established with the subspace of the tempered distributions being replaced by a certain subspace of the quasimeasures. For $p=q$, this subspace becomes a subspace of the pseudomeasures.

THEOREM 5.1. Suppose $p \leqq q, p, q \in[1, \infty]$ and that $T$ is a multiplier of type $(p, q)$. Then there exists a quasimeasure $s$ such that

$$
T f=s * f \quad\left(f \in C_{c}\right)
$$

If $p \neq \infty, L_{p}^{q}$ is isomorphic to a vector subspace of $D^{\prime}$. 
Proof. $T \mid C_{c}$ is an operator from $C_{c}$ into $M$ which commutes with convolution. Furthermore, since $T$ is continuous from $L^{p}$ into $L^{q}$, $T \mid C_{c}$ is certainly continuous for the inductive limit and vague topologies on $C_{c}$ and $M$ respectively. Hence, by Theorem 3.2 , there exists a quasimeasure $s$ with (5.1.1) holding.

Note. If $p=q$, the multipliers of type $(p, q)$ are defined by convolution with the elements of a subspace of the pseudomeasures. This may be established by use of the Riesz Convexity Theorem and the fact that $L_{2}^{2}=P(G)$. Another known result (Wendel [12]) is that $L_{1}^{1}=M_{b d}$.

5.2. In a recent research announcement [6], Figà-Talamanca has given a characterisation of $L_{p}^{p}$ as the dual of a certain space of continuous functions which tend to zero at infinity. It is possible to deduce this result from Theorem 5.1.

\section{REFERENCES}

1. N. Bourbaki, Eléments de mathématique, Topologie générale, Chapitre 9, Hermann, Paris 1958.

2. — Eléments de mathématique, Algèbre, Chapitre 2, Hermann, Paris, 1947.

3. R. E. Edwards, Convolutions as bilinear and linear operators, Canad. J. Math. 116 (1964), 275-285.

4. - Functional analysis: theory and applications, Holt, Rinehart and Winston, New York, 1965.

5. — Operators commuting with translations, Pacific J. Math. 16 (2) (1966), 259-265.

6. Alessandro Figà-Talamanca, Multipliers of p-integrable functions, Bull. Amer. Math. Soc. 70, (1964), 666-669.

7. Edwin Hewitt, and Kenneth Ross, Abstract harmonic analysis, Vol. 1, SpringerVerlag, Berlin, 1963.

8. Lars Hörmander, Estimates for translation-invariant operators in $L^{p}$ spaces, Acta Math. 104 (1960), 93-139.

9. Jean-Pierre Kahane, Transformées de Fourier des functions sommables, Proc. Int. Congr. Math. (1962), 114-131.

10. W. Rudin, Fourier analysis on groups, Interscience, New York, 1962.

11. Laurent Schwartz, Théorie des distributions, Tome I, Hermann, Paris, 1950.

12. J. G. Wendel, Left centralizers and isomorphisms of group algebras, Pacific J. Math. 2 (1952), 251-261.

Received June 18, 1965. The author wishes to thank Dr. R. E. Edwards for his help and for the suggestion to introduce the space $D$ and its dual. A generous financial grant from the Reserve Bank of Australia made this work possible.

Institute of Advanced Studies

The Australian National University

Canberra, Australia 


\section{PACIFIC JOURNAL OF MATHEMATICS}

\section{EDITORS}

\author{
H. SAMELSON, \\ Stanford University \\ Stanford, California \\ R. M. BLUMENTHAL \\ University of Washington \\ Seattle, Washington 98105
}

\author{
*J. DugundJI \\ University of Southern California \\ Los Angeles, California 90007 \\ RICHARD ARENS \\ University of California \\ Los Angeles, California 90024
}

\section{ASSOCIATE EDITORS}
E. F. BECKENBACH
B. H. NeUmanN
F. WOLF
K. YOSIDA

\section{SUPPORTING INSTITUTIONS}

\author{
UNIVERSITY OF BRITISH COLUMBIA \\ CALIFORNIA INSTITUTE OF TECHNOLOGY \\ UNIVERSITY OF CALIFORNIA \\ MONTANA STATE UNIVERSITY \\ UNIVERSITY OF NEVADA \\ NEW MEXICO STATE UNIVERSITY \\ OREGON STATE UNIVERSITY \\ UNIVERSITY OF OREGON \\ OSAKA UNIVERSITY \\ UNIVERSITY OF SOUTHERN CALIFORNIA
}

\author{
STANFORD UNIVERSITY \\ UNIVERSITY OF TOKYO \\ UNIVERSITY OF UTAH \\ WASHINGTON STATE UNIVERSITY \\ UNIVERSITY OF WASHINGTON \\ * * * \\ AMERICAN MATHEMATICAL SOCIETY \\ CHEVRON RESEARCH CORPORATION \\ TRW SYSTEMS \\ NAVAL ORDNANCE TEST STATION
}

Mathematical papers intended for publication in the Pacific Journal of Mathematics should be typewritten (double spaced). The first paragraph or two must be capable of being used separately as a synopsis of the entire paper. It should not contain references to the bibliography. Manuscripts may be sent to any one of the four editors. All other communications to the editors should be addressed to the managing editor, Richard Arens at the University of California, Los Angeles, California 90024 .

50 reprints per author of each article are furnished free of charge; additional copies may be obtained at cost in multiples of 50 .

The Pacific Journal of Mathematics is published monthly. Effective with Volume 16 the price per volume (3 numbers) is $\$ 8.00$; single issues, $\$ 3.00$. Special price for current issues to individual faculty members of supporting institutions and to individual members of the American Mathematical Society: $\$ 4.00$ per volume; single issues $\$ 1.50$. Back numbers are available.

Subscriptions, orders for back numbers, and changes of address should be sent to Pacific Journal of Mathematics, 103 Highland Boulevard, Berkeley 8, California.

Printed at Kokusai Bunken Insatsusha (International Academic Printing Co., Ltd.), No. 6, 2-chome, Fujimi-cho, Chiyoda-ku, Tokyo, Japan.

PUBLISHED BY PACIFIC JOURNAL OF MATHEMATICS, A NON-PROFIT CORPORATION

The Supporting Institutions listed above contribute to the cost of publication of this Journal, but they are not owners or publishers and have no responsibility for its content or policies.

* Paul A. White, Acting Editor until J. Dugundji returns. 


\section{Pacific Journal of Mathematics}

\section{Vol. 18, No. 3 \\ May, 1966}

William George Bade and Philip C. Curtis, Jr., Embedding theorems for commutative Banach algebras .......................... 391

Wilfred Eaton Barnes, On the $\Gamma$-rings of Nobusawa ................. 411

J. D. Brooks, Second order dissipative operators ................ 423

Selwyn Ross Caradus, Operators with finite ascent and descent ........ 437

Earl A. Coddington and Anton Zettl, Hermitian and anti-hermitian properties of Green's matrices .......................... 451

Robert Arnold Di Paola, On sets represented by the same formula in distinct consistent axiomatizable Rosser theories ................... 455

Mary Rodriguez Embry, Conditions implying normality in Hilbert space ...........................................

Garth Ian Gaudry, Quasimeasures and operators commuting with convolution ................................... 461

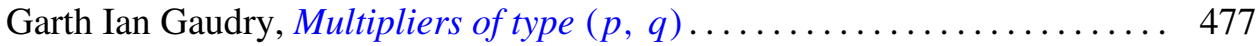

Ernest Lyle Griffin, Jr., Everywhere defined linear transformations affiliated with rings of operators .................................. 489

Philip Hartman, On the bounded slope condition ................ 495

David Wilson Henderson, Relative general position ................ 513

William Branham Jones, Duality and types of completeness in locally

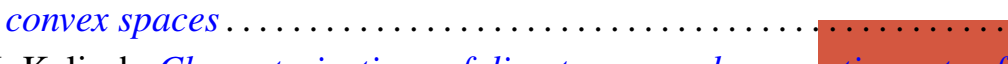

G. K. Kalisch, Characterizations of direct sums and commuting sets of Volterra operators....

Ottmar Loos, Über eine Beziehung zwischen Malcev-Algebren und Lietripelsystemen

Ronson Joseph Warne, A class of bisimple inverse semigroups . . . 